\title{
MORPHOLOGICAL VARIATIONS OF THE LUNGS: A CADAVERIC STUDY
}

\author{
Ajeevan Gautam, ${ }^{1}$ Rajib Chaulagain, ${ }^{2}$ Deepesh Dhungel ${ }^{3}$
}

${ }^{1}$ Department of Anatomy, ${ }^{2}$ Department of Oral Pathology, Chitwan Medical College, Bharatpur-13, Chitwan, ${ }^{3}$ Department of Anatomy, Institute of Medicine, Maharajgunj Medical Campus, Maharajgunj, Kathmandu, Nepal

\section{ABSTRACT}

The lungs are the organs of respiration which are situated on either side of the heart and other mediastinal contents in its pleural cavity. A fresh lung is spongy, can float in water and crepitates when handled. Lungs are important with respect to its blood circulation. The lungs are divided by fissures into lobes which facilitate movements of lobes in relation to one another. The hilum of each lung is its gateway. In the present study, we aim to assess the morphological variations of human cadaveric lungs at Chitwan Medical College (CMC). An observational study was conducted at dissection hall of anatomy department at Chitwan Medical College from September 2019 to October 2020 after taking ethical approval form Institutional Review Committee of CMC. All the intact 70 lungs present in the department were studied. Photographs of the intact lungs were taken from different surface. The lungs were porus, highly elastic and spongy in texture. On keeping lungs to water tank it got floated. We found $34(80.96 \%)$ of the studied specimen of right side had horizontal fissure present in it. The remaining 8 (19.04\%) specimens did not have horizontal fissures, while $3(5.88 \%)$ specimens had incomplete fissures. The oblique fissure was not present in $2(2.38 \%)$ of the study specimens. The left side of the study specimen has a variance of $1(4.16 \%)$. When the hilum right lung was examined, $40(95.23 \%)$ of the structure had the usual organization pattern. In the left lung, the usual pattern of organization was 21(75\%). The differences are thought to be present in the lung's fissure and hilum. The current study's findings are therapeutically important. The findings could prove beneficial to cardiovascular and thoracic surgeons.

\section{KEYWORDS}

Cadaver, Lungs, Anatomy, Variation, Fissure, Hilum

Received on: June 25, 2021

Accepted for publication: November 04, 2021

\section{CORRESPONDING AUTHOR}

Dr. Ajeevan Gautam,

Assistant Professor,

Department of Anatomy,

Chitwan Medical College,

Bharatpur-5, Chitwan, Nepal

Email: gautamaajeevan@gmail.com

Orcid No: https://orcid.org/0000-0001-7673-181X

DOI: https://doi.org/10.3126/nmcj.v23i4.42221 


\section{INTRODUCTION}

The lungs are the organs of respiration which are situated on either side of the heart and other mediastinal contents in its pleural cavity. The average weight of right lung is about 600gm and of left lung is $500 \mathrm{gm} .{ }^{1}$

A fresh lung is spongy, can float in water and crepitates. It is highly elastic so it retracts on removal from the thorax. Its surface is smooth and shiny and is separated by fine, dark lines into numerous small polyhedral domains, each crossed by numerous finer lines, indicating the areas of contact between its most peripheral lobules and the pleural surface. ${ }^{2}$

Lungs are important with respect to its blood circulation. Kidney gets $21 \%$ of the Cardiac output, ${ }^{3,4}$ but pulmonary blood flow is approximately equal to $100 \%$ of the output of the left ventricle. ${ }^{5}$

The lungs are divided by fissures into lobes which facilitate movements of lobes in relation to one another.The right lung is divided into three lobes: upper, middle and lower by oblique and horizontal fissures. The left lung is divided into two lobes: upper and lower by oblique fissure. ${ }^{6}$

The hilum of each lung contains a main bronchus, a pulmonary artery, two pulmonary veins, the pulmonary nerve plexus and lymph nodes, all enveloped by the pleural cuff: an inferior narrow extension of the cuff is known as the pulmonary ligament. The bronchus lies behind the pulmonary artery and the two veins are below and anterior. ${ }^{7}$

The location of lobes and fissures is important both physically and clinically in determining the bronchopulmonary segments. Prior awareness and anatomical knowledge of changes in lung lobes and fissures is critical for correct radiological interpretation and is crucial for cardiothoracic surgeons planning segmental resection. ${ }^{8}$ The study aimed to assess the morphological variations of the human cadaveric lung.

\section{MATERIALS AND METHODS}

This was an observational study conducted at Chitwan Medical College from September 2019 to October 2020. Ethical approval was obtained from CMC-IRC/076/077-151.

Seventy (70) isolated, properly embalmed formalin fixed cadaveric lungs preserved in the dissection hall were taken in consideration for the study. All the intact lungs present in the department were studied and the age estimation of the lungs were not done. A welldesigned proforma was used to collect the study variables. The lungs with cuts and deformity were excluded from the study. Out of 70 lungs, 42 were right lungs and 28 were left lungs. The parameters noted were number of fissures and lobes, shape of lungs and their lobes, and variations in the hilar structures. Photographs were taken as a record.

\section{RESULTS}

Both the right and left lungs were intact in the samples under investigation. The apex, base, costal, and medial surfaces of each lungs were half conical in shape. The lungs were porous, spongy, and very elastic. The floated by maintaining their lungs in a water tank. The lungs had crepitations when touched and were a dark slety grey color, which could be due to carbon particles deposited on the stroma.

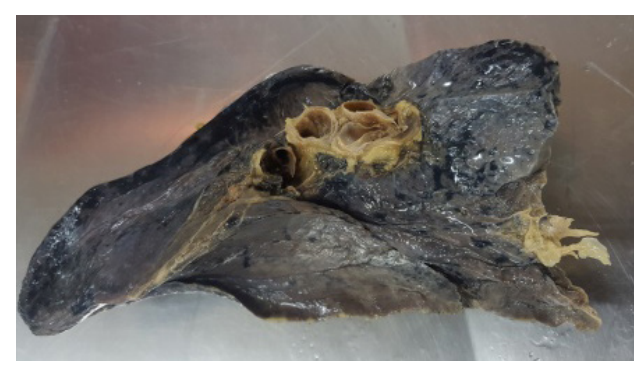

Fig. 1: Photograph with absence of horizontal fissure of right lung

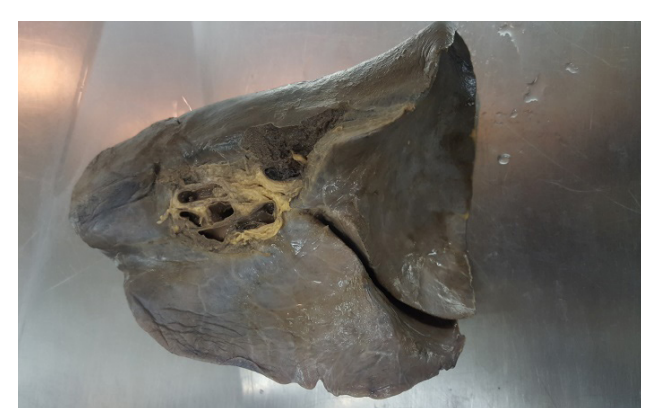

Fig. 2: Photograph of hilum with Normal Fissure

There are two fissures in the right lung. The horizontal fissure and the oblique fissure are two types of fissures. We discovered a change in typical pattern in the study that we conducted. We discovered that horizontal fissures were prevalent in $34(80.96 \%)$ of the lungs investigated. The remaining 8 (19.04\%) of the study specimens lacked a horizontal fissure, while $3(5.88 \%)$ had an incomplete fissure.

When the presence of oblique fissure was examined, $40(95.23 \%)$ of the lungs had 


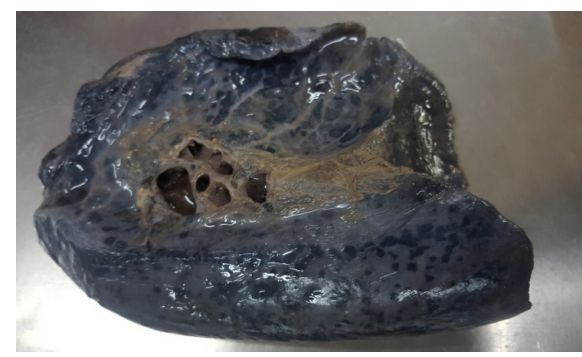

Fig. 3: Left lung without fissure

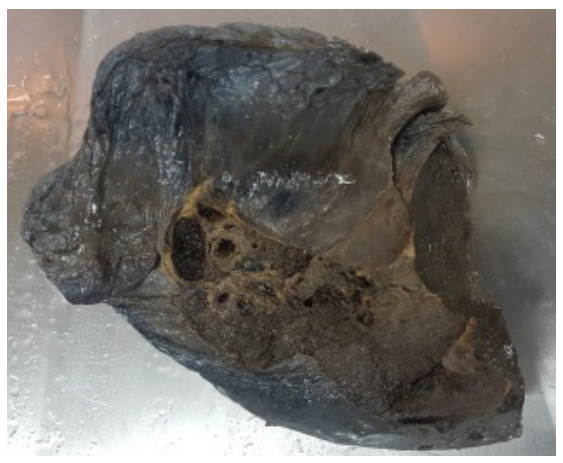

Fig. 4: Hilum of lung

complete oblique fissure. 2(4.87\%) of the investigated specimens had no oblique fissure, while 2(4.87\%) had an incomplete oblique fissure. Only an oblique fissure can be found in the left lung. It was found in all of the specimens analyzed, but one (4.16\%) showed an unique fissure pattern.

We also looked at the differences in structures at the lung's hilum. The usual arrangement pattern of the structure was found in 40 (95.23 percent) of the right lung analyzed. However, there was variation in $2(4.76 \%)$ of it. The following was a variation of the hilum's structural arrangement: In one example, there were two pulmonary arteries, whereas in another, there were four pulmonary arteries. All of the specimens analyzed had two bronchus epartial and hyparterial bronchus.

The structural arrangement of the hilum was found to vary in $7(25 \%)$ of the left lung studied. We detected 2 pulmonary arteries in $4(14.28 \%)$ of the cases and 3 pulmonary arteries in 3 $(10.71 \%)$ of the cases. On entering the hilum, the solitary bronchus with its division was visible.

\section{DISCUSSION}

The development of lung starts as multiple bronchopulmonary bud. Later the fissures that separate individual bronchopulmonary buds gets obliterated. The remaining space along the interlobar planes gives rise to major (oblique) and minor (horizontal) fissure in a fully developed lung. ${ }^{9,10}$ Defective pulmonary development is associated with the variations as encountered in fissures and lobes. ${ }^{11}$ Incomplete pulmonary fissures indicating partial fusion between lobes are common and more than half of the pulmonary fissures are incomplete. ${ }^{10}$

The detailed knowledge regarding the anatomy of fissures is of great significance for planning operative strategy for various procedures like thoracoscopic pulmonary resection or pulmonary lobectomy where incomplete fissure may be the cause of post-operative air leakage. Presence of fissures in lung enhances uniform expansion of lungs and their position might be used as a reliable landmark in specifying lesions within the lungs or thorax as well. ${ }^{13}$ Incomplete fissures usually change the pattern of collapse which is normally seen in the patients of endobronchial lesions and also contribute to the atypical appearance of pleural effusion. An incomplete major fissure causes the pseudo appearance of fluid within the fissure. ${ }^{14}$ Incomplete fissure may alter the spread of disease within lung. Pneumonia may spread to adjacent lobes via parenchymal continuation in case of incomplete fissures. ${ }^{15}$

Study conducted on morphological study of lung lobe and fissure at India demonstrated absence of horizontal fissure in $2.56 \%$ in right side. Incomplete oblique fissure was demonstrated on $10.26 \%$ and $5.26 \%$ respectively on right and left side. Oblique fissure was found missing in $2.56 \%$ of right lungs and horizontal fissure was absent in $23.08 \%$ of right lungs. ${ }^{16}$ This study documented absence of horizontal fissure in $19.04 \%$ of the right lung.

Among the lung specimen of right side with presence of horizontal fissure we found incomplete horizontal fissure on $5.88 \%$. Regarding oblique fissure it was absent in $2.38 \%$ and $14.28 \%$ on right and left side respectively. The findings of their study are against the finding of ours, this difference might be because of difference in sample size.

Nene et al in their study titled lung lobes and fissure documented absence of right oblique and horizontal fissure in $2 \%$ and $14 \%$ of the study specimen. They also documented that left oblique fissure present in all the specimen ${ }^{11}$. Our study also showed absence of right oblique and horizontal fissure in $2.38 \%$ and $19.04 \%$ respectively. This similarity might be because of study being conducted in similar region.

The study including 110 cadaveric lung specimens of south Indian population was conducted for the structural arrangement on the bronchial pattern. They found $83.9 \%$ of the right lung exhibited normal arrangement 
of structure in the hilum. They observed two pulmonary arteries in $8.9 \%$ and four pulmonary vein in $1.8 \%$ of the right lung. Similarly, $51.8 \%$ of the left lung exhibited normal pattern of arrangement in the hilum. Two pulmonary was recorded in 5.5\%. ${ }^{16}$ Our recording showed $97.06 \%$ of the hilum of right lung had normal pattern of arrangement. We found 2 pulmonary arteries in $2.1 \%$ of the specimen and 4 pulmonary in $0.84 \%$ of the specimen. Similarly, $75 \%$ of the left lung hilum showed normal pattern of arrangement. ${ }^{2}$ pulmonary artery was found in $14.28 \%$ and 3 pulmonary arteries in $10.71 \%$ of the studied hilum. The finding of both the study shows near similarity, this might be because of similar sample size and similar region of study.

Medlar $^{17}$ in his study reported $62.3 \%$ lung specimen with absence or incomplete horizontal fissure of right lung. They also recorded 25.6$30 \%$ and $10.6-18 \%$ with incomplete oblique fissure in right and left side respectively. The findings of their study is at odds with our findings. Our findings shows $19.04 \%$ without horizontal fissure and $5.88 \%$ with incomplete fissure in right side and $4.16 \%$ with unique fissure in left side. This difference might be because of different sample size, region and time of the study.

The findings of the current study could prove beneficial to radiologist, cardiovascular and thoracic surgeons for lung related thoracic surgeries and future researchers working with morphological variations. It also has an academic interest all medical persons. The morphological classification of the lung will also aid in re-evaluation, resulting in more accurate assessments of individual prognoses and better predictions of response to specific treatment regimens for cancer patient. ${ }^{18}$ The present study will provide the database for the variant anatomy of the arrangement of structures of the lung. We suggest for more studies with more sample size which may give different picture for the variation.

\section{ACKNOWLEDGEMENTS}

We would like to thank the CMC IRC for ethical clearance and whole heartedly thank the administration and department without whom the study would not have been possible.

Conflict of interest: None

Source of research fund: None

\section{REFERENCES}

1. Kulkarni NV. Clinical Anatomy: A problem solving approach $2^{\text {nd }}$ edition 219-28: 208-28.

2. Bannister LH, Berry M, Collins P et al. Grays Anatomy: The anatomical basis of medicine and surgery.38th edition 1657-62.

3. Gautam A, Khan GA, Timilsina S, Dhungel D, Sah SK. Variation in formation of renal artery. J Coll Med Sci Nep 2020; 16: 41-3. DOI: 10.3126/jcmsn. v16i1.24472.

4. Levitzky MG. Pulmonary physiology $9^{\text {th }}$ edition Mc Graw Hill Lange.

5. Hall JE. Guyton and Hall Textbook of Medical Physiology.12th Edison.

6. George BM, Nayak SB, Marpalli S. Morphological variations of the lungs: a study conducted on Indian cadavers. Anat Cell Biol 2014; 47: 253-8.

7. Datta AK. Essentials of human anatomy, part $1-$ thorax and abdomen, 3rd edn. Current Books International, Calcutta: 1994.

8. Thapa P, Desai SP. Morphological variation of human lung fissures and lobes: An anatomical cadaveric study in North Karnataka, India. Ind $J$ Health Sci Biom Res 2016; 9: 284-7.

9. Larsen WJ. Human Embryology. New York: Churchill Livingstone; 1993; 111-30.

10. Nene AR, Gajendra KS, Sarma MVR. Lung lobesand fissures: a morphological study. Anatomy- Inter J Experim Clinic Anat 2011; 30-7.
11. Bhimadevi N, Rao NB, Sunitha V. Morphological variations of lung- A cadaveric study in north coastal Andhra Pradesh. Inter J Biolog Medi Res 2011; 2: 1149-52.

12. Kent EM, Blades B. The surgical anatomy of the pulmonary lobes. J Thoracic Surg 1942; 12: 1830.

13. Meenakshi S, Manjunath KY, Balasubramanyam V. Morphological variations of lung fissures and lobes. Ind J Chest Dis Allied Sci 2004; 46: 179-82.

14. Ariyurek OM, Gulsum M, Demirkazik FB. Accessory fissures of the lung: evaluation by high-resolution computed tomography. Eur Radiol 2001; 11: 2449-53.

15. Anjankar V, Wankhede KP, Mangalgiri A. Morphological study of lung lobes and fissure: Anatomical basis of surgical and imaging technique. Int'l J Anat Res 2017; 5: 3447-50. DOI: https://dx.doi.org/10.16965/ijar.2016.502

16. Murlimanju BV, Massand A, Madhyastha S, Pai MV, Prabhu LV, Saralaya VV. Anatomical variations of the arrangement of structures at the pulmonary hilum: a cadaveric study. Surg Radiol Anat 2017; 39: 51-6. DOI 10.1007/s00276016-1716-8.

17. Medler E. Morphological variations inter lobar fissures. Am J Roentoenol Radium Ther 1947; 57: 723-5.

18. Peterson I. The morphological and molecular diagnosis of lung cancer. Dtsch Arztebl Int'l 2011; 108: 525-31. doi: 10.3238/arztebl.2011.0525. 